\title{
Política Nacional de Atenção Básica 2017: retrocessos e riscos para o Sistema Único de Saúde
}

\author{
National Policy of Primary Healthcare 2017: setbacks and risks to the \\ Unified Health System
}

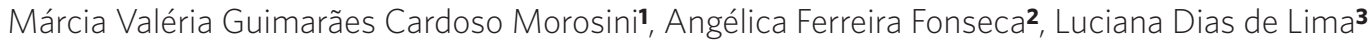

1 Fundação Oswaldo Cruz (Fiocruz), Escola Politécnica de Saúde Joaquim Venâncio (EPSJV)

- Rio de Janeiro (RJ), Brasil. mvgcmorosini@gmail.com

2 Fundação Oswaldo Cruz (Fiocruz), Escola Politécnica de Saúde Joaquim Venâncio (EPSJV) - Rio de Janeiro (RJ), Brasil. afonseca@fiocruz.br

3 Fundação Oswaldo Cruz (Fiocruz), Escola Nacional de Saúde Pública Sérgio Arouca (Ensp), Programa de Pós-Graduação em Saúde Pública - Rio de Janeiro (RJ), Brasil. luciana@ensp.fiocruz.br
RESUMO O artigo discute os significados e as implicações das mudanças introduzidas pela Política Nacional de Atenção Básica 2017, que promovem a relativização da cobertura universal, a segmentação do acesso, a recomposição das equipes, a reorganização do processo de trabalho e a fragilização da coordenação nacional da política. Argumenta-se que sua revisão indica sérios riscos para as conquistas obtidas com o fortalecimento da Atenção Primária à Saúde no Brasil. Na conjuntura atual de fortalecimento da ideologia neoliberal, tais modificações reforçam a subtração de direitos e o processo de desconstrução do Sistema Único de Saúde em curso no País.

PalAVRAS-CHAVE Atenção Primária à Saúde. Saúde da família. Política pública. Política de saúde.

ABSTRACT The article discusses the meanings and implications of the changes introduced by the National Policy of Primary Healthcare 2017, which promote the relativization of universal coverage, the segmentation of access, the recomposition of the teams, the reorganization of the work process and the weakening of the national policy coordination. It is argued that its review indicates serious risks to the achievements obtained with the strengthening of the Primary Health Care in Brazil. In the current conjuncture of strengthening neoliberal ideology, these changes reinforce the subtraction of rights and the process of deconstruction of the Unified Health System in progress in the Country.

KEYWORDS Primary Health Care. Family health. Public policy. Health policy. 


\section{Introdução}

Transcorridos 27 anos desde a promulgação das Leis $n^{\circ} 8.080$ e $n^{\circ}$ 8.142, de 1990, é possível dizer que, mesmo com dificuldades e lacunas, foram as políticas direcionadas para o fortalecimento da Atenção Primária à Saúde (APS) no Brasil que mais favoreceram a implantação dos princípios e diretrizes do Sistema Único de Saúde (SUS).

Expressas por meio de documentos específicos, as Políticas Nacionais de Atenção Básica (PNAB) ${ }^{1-3}$ tiveram papel fundamental nesse processo, permitindo inflexões importantes, principalmente no modelo de atenção e na gestão do trabalho em saúde nos municípios. Isso ocorreu de modo articulado à introdução dos mecanismos de financiamento que desempenharam papel indutor na sua adoção como eixo estruturante da organização das ações e serviços de saúde ${ }^{\mathbf{4}, 5}$.

Em setembro de 2017, foi publicada uma nova $\mathrm{PNAB}^{3}$, que suscitou a crítica de organizações historicamente vinculadas à defesa do SUS, como a Associação Brasileira de Saúde Coletiva (Abrasco), o Centro Brasileiro de Estudos de Saúde (Cebes) e a Escola Nacional de Saúde Pública (Ensp). Em nota conjunta, as três instituições denunciaram, entre outras coisas, a revogação da prioridade dada à Estratégia Saúde da Família (ESF) na organização do SUS com a provável perda de recursos para outras configurações da Atenção Básica (AB), em um contexto de retração do financiamento da saúde. Demonstraram preocupação com retrocessos em relação à construção de uma APS integral, que vinha direcionando o modelo de $\mathrm{AB}$ baseado na ESF 6 .

Este artigo teve como objetivo analisar as alterações nas diretrizes da $\mathrm{AB}$ promovidas no texto da PNAB 2017, tomando como parâmetros a PNAB 2011 e os princípios da universalidade e da integralidade da atenção à saúde, na perspectiva de uma APS forte e de uma rede integrada de atenção à saúde no SUS. Os temas analisados foram: a cobertura; a posição relativa da ESF; a configuração das equipes; e a organização dos serviços.

Inicialmente, foram identificados alguns marcos instituintes da $\mathrm{AB}$ no Brasil e suas contribuições para a configuração do SUS. Na sequência, procurou-se situar o contexto de formulação dessa nova política e discutir prováveis riscos e retrocessos das alterações propostas para o SUS.

\section{Apontamentos sobre a trajetória da política de Atenção Básica no Brasil}

Em 1994, a criação do Programa Saúde da Família (PSF) permitiu ampliar a cobertura em saúde, em um movimento inicialmente voltado apenas para a parte da população brasileira em situação social mais vulnerável. Configurou-se um modo de compor a equipe e de organizar o processo de trabalho, com base territorial e responsabilidade sanitária, referências sustentadas pelas sucessivas políticas.

Com a Norma Operacional Básica do SUS de 1996 (NOB/96), o PSF assumiu a condição de estratégia de reorientação da APS, em substituição às modalidades tradicionais. A NOB/96 instituiu os componentes fixo e variável do Piso da Atenção Básica (PAB) e estabeleceu incentivos financeiros aos municípios que adotassem o Programa de Agentes Comunitários de Saúde (Pacs) e o PSF, tornando automática e regular a transferência de recursos federais para o financiamento desses programas ${ }^{7}$.

Essa priorização teve repercussões concretas, e, em 1998, foi estabelecido o primeiro Pacto de Indicadores da Atenção Básica ${ }^{8}$, processo que se renovou periodicamente por meio da negociação intergestores de metas para a avaliação e o monitoramento da $\mathrm{AB}$ no SUS. Desdobrou-se, também, em outros dispositivos de fortalecimento da $\mathrm{AB}$, como, por exemplo, a criação do Sistema de Informação da Atenção 
Básica (Siab), também em 1998, substituindo o Sistema de Informação do Programa de Agente Comunitário de Saúde (Sipacs).

Ainda visando à reorientação do modelo de atenção, foi criado, em 2002, o Projeto de Expansão e Consolidação do Saúde da Família (Proesf), voltado para os municípios com mais de 100 mil habitantes, explicitando a compreensão da saúde da família como uma estratégia viável não apenas nas pequenas cidades e no meio rural, onde se implantou originalmente. No âmbito do Proesf, foi criada, em 2005, a Avaliação para a Melhoria da Qualidade (AMQ), que instituiu uma metodologia de avaliação em diversos níveis: gestores, coordenadores, unidades de saúde e Equipes da Saúde da Família (EqSF), com o propósito de qualificação da AB por meio da avaliação?.

A agenda política de fortalecimento da APS por meio da ESF consolidou-se gradativamente e, em 2006, tornou-se uma das dimensões prioritárias do Pacto pela Vida ${ }^{10}$. Naquele mesmo ano, foi publicada a PNAB ${ }^{1}$, revisada em $2011^{2}$, buscando preservar a centralidade da ESF para consolidar uma APS forte, ou seja, capaz de estender a cobertura, prover cuidados integrais e desenvolver a promoção da saúde, configurando-se como porta de entrada principal do usuário no SUS e eixo de coordenação do cuidado e de ordenação da Rede de Atenção à Saúde (RAS). Esse processo envolveu um amplo escopo de ações, mobilizando instituições e sujeitos sociais para responder aos desafios colocados para a formação de trabalhadores, a organização do processo de trabalho, as interações com a comunidade, a compreensão do território e as relações entre os entes federados.

Segundo Magalhães Júnior e Pinto"1, há, pelo menos, dois indicadores importantes da centralidade dada à PNAB no âmbito federal das políticas de saúde. São eles: o aumento superior a $100 \%$ dos recursos repassados aos municípios para o financiamento da $\mathrm{AB}$, entre 2010 e 2014; e a aplicação de recursos para a qualificação e a ampliação da estrutura das unidades, por meio de um projeto específico, o Requalifica SUS, lançado em 2011.

Também em 2011, foi instituído o Programa Nacional de Melhoria do Acesso e da Qualidade da Atenção Básica (PMAQ$\mathrm{AB}$ ), que incorporou elementos da AMQ e ampliou as vertentes de avaliação, tendo como finalidade a certificação das EqSF. Esse Programa permitiu vincular formas de transferência de recursos do $\mathrm{PAB}$ variável aos resultados provenientes da avaliação, constituindo-se em um mecanismo de indução de novas práticas.

Em termos de cobertura, dados disponibilizados pelo Ministério da Saúde (MS) indicam que a ESF alcançava $58 \%$ da população, em outubro de 2017, e sabe-se que essa cobertura chegou a atingir $100 \%$ em alguns municípios. Tudo isso mediante novos serviços, modalidades e arranjos de equipes multiprofissionais, com destaque para as equipes ampliadas pela saúde bucal e pelos Núcleos de Apoio à Saúde da Família (Nasf). Mesmo reconhecendo a persistência de problemas no acesso, na qualidade e na continuidade da atenção, diversos estudos sugerem avanços decorrentes das políticas de APS na redução de internações evitáveis e dos gastos hospitalares $^{12}$, e para a melhoria das condições de vida e saúde da população brasileira' ${ }^{\mathbf{3}, 14}$.

\section{Notas sobre a conjuntura e o texto da Política Nacional de Atenção Básica 2017}

Presenciou-se, recentemente, a reorganização das forças políticas conservadoras no Brasil, o que resultou no impedimento de Dilma Rousseff e na condução do seu vice Michel Temer à Presidência. Ao mesmo tempo, observa-se o rápido fortalecimento de uma pauta antidemocrática e autoritária, orientada para o aprofundamento da mercantilização dos direitos sociais brasileiros.

Seguiu-se a aprovação de medidas ditas 
'racionalizantes', sob a justificativa da necessidade de enfrentar o desequilíbrio fiscal, atribuído ao descontrole das contas públicas decorrentes de 'políticas paternalistas', que teriam agravado a crise econômica. A orientação é modificar a destinação dos recursos do fundo público, limitando as políticas sociais, promovendo a redução da dimensão pública do Estado e ampliando a participação do setor privado. Do mesmo modo, opera-se uma ofensiva contra a classe trabalhadora, atingindo conquistas fundamentais, como os direitos trabalhistas e previdenciários. Trata-se de um conjunto de reformas supressoras de direitos sociais, em uma represália sem proporções ainda calculadas, do capital contra o trabalho.

Entre as alterações legislativas que viabilizam esse processo, destaca-se a promulgação da Emenda Constitucional no 95/2016, conhecida como a emenda do 'Teto dos Gastos', que congela por 20 anos a destinação de recursos públicos e produz efeitos nas diversas políticas, especificamente no financiamento do SUS.

Essas medidas incidem sobre uma relação frágil entre o SUS e a sociedade brasileira, e caminham em paralelo ao fortalecimento ideológico do setor privado como alternativa de qualidade para o atendimento das necessidades de saúde. Conforma-se, assim, o terreno propício para dar prosseguimento à desconstrução do SUS, cujo financiamento jamais alcançou um patamar de suficiência e estabilidade, ao passo que as empresas privadas de planos de saúde sempre foram objeto de fortalecimento, por meio da destinação de incentivos financeiros contínuos ${ }^{15}$.

Nessa conjuntura, as tendências que orientavam a revisão da PNAB 2011 já vinham sendo anunciadas, pelo menos, desde outubro de 2016, quando foi realizado o VII Fórum Nacional de Gestão da Atenção Básica, cujos indicativos para tal revisão foram publicados em um documento-síntese ${ }^{\mathbf{1 6}}$. Ali, já se apresentava uma perspectiva regressiva, especialmente preocupante, considerando-se a correlação de forças muito desfavorável aos que defendem a saúde como um direito universal ${ }^{17}$.

Desde então, as notícias sobre a revisão da PNAB foram se intensificando, mas sua produção foi pouco divulgada oficialmente e sua discussão manteve-se em espaços restritos, como a Comissão Intergestores Tripartite (CIT) e as reuniões entre os técnicos do MS. Em 27 de julho de 2017, o texto preliminar foi apresentado na CIT e encaminhado para consulta pública por dez dias. Apesar do curto prazo, a consulta recebeu mais de 6 mil contribuições, sem que tenham gerado, entretanto, mudanças expressivas no texto original ou tenham sido divulgados os seus resultados. A nova PNAB foi aprovada em 30 de agosto de 2017, pouco mais de um mês após tornar-se oficialmente pública. Uma característica marcante do texto da nova $\mathrm{PNAB}^{3}$ é a explicitação de alternativas para a configuração e implementação da $\mathrm{AB}$, traduzindo-se em uma pretensa flexibilidade, sustentada pelo argumento da necessidade de atender especificidades locorregionais.

Supostamente, amplia-se a liberdade de escolha dos gestores do SUS, o que poderia ser visto como positivo por responder às demandas de um processo de descentralização mais efetivo. Entretanto, esse processo só se completaria com a transferência de recursos necessários à autonomia de gestão pelos municípios, e com os mecanismos de controle social e participação popular. A presente análise não valida o raciocínio otimista, justamente porque é fruto de uma leitura informada pela atual conjuntura, que indica limites rigorosos, a partir dos quais essa política e suas possibilidades se realizarão.

Pode-se dizer que o discurso da PNAB se constrói de modo ambivalente, incorporando verbos como sugerir e recomendar, que retiram o caráter indutor e propositivo da política e expressam a desconstrução de um compromisso com a expansão da saúde da família e do sistema público. Entende-se, ainda, que essa estrutura de texto tem o propósito de blindá-lo à crítica, tornando suas proposições de mais fácil assimilação, afinal, 
a partir do que está escrito, diversas opções seriam possíveis. Esta ambivalência é um recurso que permite omitir escolhas prévias (ideológicas), que parecem determinar o processo de revisão da PNAB no momento político atual. Tais escolhas remetem a uma concepção de Estado afinada com a racionalidade neoliberal, que aponta para o sentido inverso a uma maior presença do Estado, requerida para a continuidade do SUS como projeto e da $\mathrm{AB}$ como estratégia principal para a garantia da saúde como direito universal.

Feitas essas considerações mais gerais, apresenta-se a discussão de elementos específicos presentes na nova PNAB, inventariando os riscos potenciais detectados para o SUS e seus princípios.

\section{Relativização da cobertura}

Como ressaltado, a universalidade é um princípio estruturante da atenção à saúde no âmbito do SUS, que, aliada à integralidade, tem distinguido a PNAB de conformações simplificadas e focalizantes de APS. Baseadas nesses princípios, as PNAB 2006 e 2011 vinham projetando a expansão da ESF, nas duas últimas décadas. Considerando este movimento, o tema da cobertura da $\mathrm{AB}$ destaca-se como um importante indicador da intencionalidade da PNAB 2017.

Retrospectivamente, percebe-se que, entre os itens necessários à implantação da ESF nas PNAB 2006 e 2011, encontrava-se uma única referência à cobertura universal. Ela se construiu de forma mediada, representada pela relação entre o número previsto de Agentes Comunitários de Saúde (ACS) por equipe e a cobertura de $100 \%$ da população cadastrada. Nessas PNAB, constava que, para a implantação de EqSF, seria necessário um número de ACS suficiente para cobrir $100 \%$ da população cadastrada, com um máximo de 750 pessoas por ACS e de 12 ACS por EqSF. A PNAB 2011 acrescentou o alerta de que não se deveria ultrapassar o limite máximo de moradores vinculados a cada equipe, evidenciando a preocupação com as condições que poderiam diretamente afetar a qualidade da atenção.

No texto da PNAB 2017, anterior à consulta pública, não há nenhuma referência à cobertura universal. A referência a $100 \%$ de cobertura é retomada no texto publicado, porém restrita a certas áreas:

Em áreas de risco e vulnerabilidade social, incluindo de grande dispersão territorial, o número de ACS deve ser suficiente para cobrir 100\% da população, com um máximo de 750 pessoas por agente, considerando critérios epidemiológicos e socioeconômicos ${ }^{3(71)}$.

A flexibilização da cobertura populacional está relacionada também aos parâmetros da relação equipe/população apresentados no item 'Funcionamento'. Ali se lê que a população adscrita recomendada por equipe de $\mathrm{AB}$ e EqSF é de 2 mil a 3,5 mil pessoas. Entretanto, neste mesmo item, de acordo com as especificidades do território, prevê-se também a possibilidade de "outros arranjos de adscrição"3(70), com parâmetros populacionais diferentes, que podem ter alcance "maior ou menor do que o parâmetro recomendado" ${ }^{3(70)}$. A decisão a esse respeito fica a cargo do gestor municipal, em conjunto com a equipe de $\mathrm{AB}$ e o Conselho Municipal ou Local de Saúde, com a ressalva de que fica assegurada a qualidade do cuidado.

Na PNAB 2011 havia a seguinte indicação: "quanto maior o grau de vulnerabilidade, menor deverá ser a quantidade de pessoas por equipe"2(55). Segundo essa redação, o critério de flexibilização de parâmetros populacionais apontava claramente a intenção de favorecer aqueles que apresentassem maior necessidade de atenção. $\mathrm{O}$ mesmo não pode ser dito em relação à nova PNAB, que, mais uma vez, se descompromete a adotar parâmetros que favoreçam um processo de atenção progressivamente mais qualificado. Ao desconsiderar a relação entre quantidade 
e qualidade, a afirmação de que deve ser assegurada a qualidade do cuidado torna-se mera retórica.

A cobertura é igualmente relativizada por meio da indefinição do número de ACS, uma vez que a PNAB 2017 indica que

o número de ACS por equipe deverá ser definido de acordo com base populacional (critérios demográficos, epidemiológicos e socioeconômicos), conforme legislação vigente ${ }^{3(71)}$.

Deste modo, pode-se compor equipes com apenas um ACS. Quando uma política, simultaneamente, torna indefinido o número de ACS por equipes e flexibiliza os parâmetros de cobertura, reforça-se o risco de serem recompostas barreiras ao acesso à saúde de parte da população. Lembre-se, aqui, que o ACS é um trabalhador que deveria ser o 'elo' entre os serviços de saúde e a população, contribuindo para facilitar o acesso e proporcionar uma relação estável e contínua entre a população e os serviços de APS. Tal formulação compromete, também, um conjunto de processos já instituídos na ESF, que se estruturam por meio da presença constante de um trabalhador da saúde no território. Entre esses processos, destacam-se a escuta e a percepção de problemas e necessidades que poderiam ser invisíveis aos serviços, bem como a identificação e a criação de possibilidades de intervenção, dadas a partir de seus conhecimentos sobre a dinâmica da vida no território.

Essas alterações articuladas abrem um precedente inédito na história da PNAB, que desestabiliza o compromisso da política com a universalidade da atenção à saúde no SUS.

\section{A segmentação do cuidado: padrões essenciais e ampliados de serviços}

Pode-se dizer que, além da universalidade, outro princípio com o qual a PNAB 2017 se descompromete é o da integralidade. Isto se dá, principalmente, por meio da definição de padrões diferenciados de ações e cuidados para a $\mathrm{AB}$, novamente, sob o argumento das condições ou especificidades locais. Estes padrões distinguem-se entre 'essenciais' e 'ampliados'.

Os padrões essenciais são 'as ações e os procedimentos básicos', que deveriam ser garantidos pelas equipes em todo o País. Embora sejam apresentados como condições básicas de acesso à atenção à saúde, e de qualidade do cuidado oferecido, a própria segmentação os remete à ideia de mínimos. Assim, o termo ‘básico' se esvazia do sentido que é tão caro a alguns autores da literatura sobre APS no Brasil, isto é, como distintivo do compromisso da $\mathrm{AB}$, de ser o primeiro nível de acesso a um sistema universal, que abrange uma rede integral e complementar de atenção à saúde, capaz de resolver $80 \%$ dos problemas de saúde da população.

Os padrões ampliados correspondem a

ações e procedimentos considerados estratégicos para se avançar e alcançar padrões elevados de acesso e qualidade na $A B$, considerando as especificidades locais e decisão da gestão ${ }^{3(70)}$.

Entretanto, tais padrões são apenas recomendados. A PNAB projeta um compromisso que se limita aos padrões essenciais, que, como já advertido, tendem a se configurar em torno de cuidados mínimos, recuperando a concepção de APS seletiva.

Não há nenhum conteúdo especificado que possibilite conhecer e analisar a que corresponderiam tais padrões. Não se sabe quais procedimentos e ações integram o padrão básico e, portanto, mantém-se a incerteza sobre a capacidade de esse padrão atender as necessidades de saúde que deveriam ser contempladas na APS. Em relação ao padrão ampliado, cabe questionar: existem ações e cuidados que integram a $\mathrm{AB}$ hoje, e que podem ser negligenciados a 
ponto de constituírem um padrão opcional, ou seja, apenas recomendável? A análise empreendida conclui o inverso. Este é um modo de consentir com o aprofundamento das desigualdades e a segmentação do acesso e do cuidado que marcam a APS em diversos países da América Latina, como apontam Giovanella et al. ${ }^{14}$.

$O$ risco colocado, pela diferenciação entre os serviços 'essenciais' e 'ampliados', é de que se retome a lógica da seletividade com diretrizes que reforcem a segmentação e a fragmentação dos serviços e das ações de saúde no SUS, a partir da APS. Segundo Conill, Fausto e Giovanella ${ }^{18}$, a segmentação e a fragmentação são categorias fundamentais para a compreensão dos problemas dos sistemas de saúde. Estão relacionadas à garantia dos direitos sociais e são muito sensíveis aos problemas de financiamento público, com efeitos restritivos às possibilidades de acesso aos demais níveis de atenção e à constituição de redes integradas de atenção à saúde ${ }^{19}$.

A segmentação propugnada pela PNAB deve ser examinada de modo articulado à racionalidade, aos interesses e à perspectiva de sistema de saúde enunciada pelo atual ministro da saúde, Ricardo Barros, segundo a qual seria desejável compreender os setores público e privado suplementar como partes de um todo único. Neste sentido, ampliam-se as condições para que a saúde suplementar passe a integrar o sistema de serviços de saúde, desta vez diretamente impulsionado pelo desenho das políticas públicas.

A base argumentativa para a segmentação do cuidado, na nova PNAB, deturpa a ideia de especificidades territoriais que antes justificavam e davam consistência ao princípio da equidade, este que remete à obrigação ético-política de se estabelecerem parâmetros e processos visando à superação de desigualdades historicamente produzidas na sociedade brasileira, de modo a revelar e interferir sobre as condições que as produzem. Contrariamente, esta segmentação do cuidado traz as bases para o desenvolvimento de um SUS seletivo, que universaliza mínimos e estratifica padrões de atenção, justificados por situações precárias, cuja superação não está no horizonte de compromissos das políticas públicas projetadas no atual contexto. São sentidos opostos de utilização de uma noção - o território - que se fortaleceu no processo de consolidação da saúde da família como estratégia de ordenamento da AB no Brasil. Daí, o seu apelo positivo. Mais uma vez, trata-se da apropriação e ressignificação negativa de uma ideia-chave para os defensores da APS forte, que contribui para confundir o leitor quanto às intenções da nova PNAB.

Entretanto, a desconstrução do compromisso com a universalidade e com a integralidade parece fazer parte de um quadro mais amplo, que não nos permite ilusões. O horizonte imediato do setor da saúde, na perspectiva das forças políticas hegemônicas, revela-se em uma fala proferida pelo ministro Barros sobre o tema 'Gestão transformadora para a saúde pública', em um encontro com líderes empresariais ocorrido em 2016:

\footnotetext{
Queremos mais recursos para a saúde e, como estamos nessa crise fiscal, se tivermos planos acessíveis com modelos de que a sociedade deseje participar, teremos $\mathrm{R} \$ 20$ [biIhões] ou $\mathrm{R} \$ 30$ bilhões a mais de recursos, que serão colocados para atendimento de saúde. Isso vai aliviar nosso sistema, que está congestionado ${ }^{20(1)}$.
}

Com o intuito de viabilizar esse propósito, o MS criou um Grupo de Trabalho (GT) para elaborar um projeto que possibilite a oferta de planos de saúde com número de serviços inferior ao definido como cobertura mínima pela Agência Nacional de Saúde Suplementar (ANS). A adesão a esses planos 'populares' de saúde - entendidos aqui como simplificados - seria voluntária.

Como se gera a 'adesão voluntária' ao setor privado? A resposta que esta reflexão busca oferecer pode parecer óbvia ou redundante: se 
produz adesão ao setor privado por exclusão do setor público. Entretanto, não é óbvio compreender que a exclusão do setor público deve ser ativamente produzida e se faz por meio da restrição ao acesso, associada à baixa qualidade dos serviços. É esta combinação que acarreta a evasão de parte das classes populares ou a não adesão da classe média ao SUS.

Compreende-se que estão em curso três eixos de ação: a) definir padrões mínimos e ampliados para a AB; b) estabelecer uma "regulação que permita menos cobertura e menos custo"20; e c) colocar no mercado planos baseados na oferta de um rol mínimo do mínimo 20,21 de serviços. Articuladas, estas ações integram um processo que, pela exclusão do SUS, pode gerar clientela para os planos privados e viabilizar planos incapazes de atender às necessidades de saúde. Neste cenário, a nova PNAB tende a servir como plataforma para o avanço de políticas que aprofundem tais possibilidades.

A naturalização da ingerência do setor privado no SUS, expressa por pensamentos tais como 'isso já acontece', contribui para ofuscar o fato de que a $A B$ vinha se constituindo como um contraponto a essa realidade. Uma vez sustentado o movimento de expansão e qualificação da ESF, a $\mathrm{AB}$ tenderia a concretizar, a médio e longo prazo, a experiência do acesso à atenção à saúde, efetivamente pública. Esta é a aposta que está sendo suspensa ou interrompida.

\section{$O$ reposicionamento da Estratégia Saúde da Família e a retomada da Atenção Básica tradicional}

Em relação ao papel da ESF, o texto da PNAB 2017 apresenta uma posição, no mínimo, ambígua. Ao mesmo tempo em que mantém a ESF como prioritária no discurso, admite e incentiva outras estratégias de organização da $\mathrm{AB}$, nos diferentes territórios:
Art. 4 - A PNAB tem na saúde da família sua estratégia prioritária para expansão e consolidação da Atenção Básica. Contudo, reconhece outras estratégias de organização da Atenção Básica nos territórios, que devem seguir os princípios, fundamentos e diretrizes da Atenção Básica e do SUS descritos nesta portaria, configurando um processo progressivo e singular que considera e inclui as especificidades locorregionais, ressaltando a dinamicidade do território ${ }^{3(68)}$.

Essa ambiguidade torna-se mais visível quando se analisam, em conjunto, certos elementos dessa política. Destacam-se, principalmente, alterações nas regras de composição profissional e de distribuição da carga horária dos trabalhadores nas equipes de $\mathrm{AB}$.

A presença dos ACS não é requerida na composição mínima das equipes de $\mathrm{AB}$, diferentemente do que acontece na ESF. Considerando as recentes conquistas desses trabalhadores, em relação aos vínculos empregatícios e à definição do piso salarial da categoria, entende-se que esta é uma possibilidade que desonera financeiramente a gestão municipal, tornando-se extremamente atraente no contexto de redução de recursos já vivenciado, e cujo agravamento é previsto.

A presença desse trabalhador e a continuidade, com regularidade, das ações por ele desempenhadas nunca estiveram tão em risco. O prejuízo recai principalmente sobre as ações educativas e de promoção da saúde, pautadas pela concepção da determinação social do processo saúde-doença e da clínica ampliada, que configuram bases importantes para a reestruturação do modelo de atenção à saúde.

Em relação à carga horária, determina-se o retorno da obrigatoriedade de 40 horas para todos os profissionais da ESF, inclusive os médicos, cuja carga horária havia sido flexibilizada na PNAB 2011. Esta alteração retoma condições estabelecidas desde a implantação do PSF, que são consideradas 
positivas para favorecer o vínculo entre profissional e usuário, e potencializar a responsabilidade sanitária das equipes e a continuidade do cuidado. Entretanto, sabe-se que a flexibilização da carga horária dos médicos de 40 para 20 horas buscava equacionar a dificuldade de fixação desse profissional nas equipes, um problema que persiste.

Diferentemente do que é previsto para a ESF, a carga horária projetada para as equipes de $A B$ (médico, enfermeiro, auxiliar ou técnico de enfermagem) deve atender às seguintes orientações: a soma da carga horária, por categoria, deve ser, no mínimo, de 40 horas; a carga horária mínima de cada profissional deve ser de 10 horas; o número máximo de profissionais por categoria deve ser três.

Operacionalmente, são inúmeros os arranjos possíveis para a composição das equipes de AB. Para fins de ilustração, uma equipe pode ser composta por três médicos, dos quais dois devam ter cargas horárias de 20 horas e um de 10 horas; três enfermeiros, com 40 horas cada; um auxiliar ou técnico de enfermagem, com 40 horas; e nenhum ACS. Isto significa que, pelo menos, três fatores tornam mais atraente a composição de equipes no modelo de $\mathrm{AB}$ tradicional: contam com menos profissionais do que a ESF e, portanto, podem ter um custo mais baixo; são mais fáceis de organizar, em função da flexibilidade da carga horária; e, agora, são também financeiramente apoiadas. É importante relembrar que a referência de população coberta também foi flexibilizada.

Embora essas alterações possam ser lidas como meros instrumentos de gestão, visando à redução de custos, cabe questionar, ainda, os seus possíveis efeitos sobre o modelo de atenção à saúde. Tal formatação de equipes tende a fortalecer a presença de profissionais cuja formação permanece fortemente orientada pelo modelo biomédico, curativo e de controle de riscos individuais. Neste sentido, antes mesmo que a ESF tenha avançado significativamente na transformação do modelo de atenção, a PNAB 2017 representa uma regressão em relação a esse propósito, criando as condições para a expansão da $\mathrm{AB}$ tradicional e fortalecendo o binômio queixa-conduta.

\section{Integração das atribuições ou fusão dos Agentes Comunitários de Saúde e dos Agentes de Combate às Endemias?}

Desde a realização do já mencionado VII Fórum de Gestão da Atenção Básica, em outubro de 2016, pelo MS, com o propósito de produzir subsídios para a revisão da PNAB, tem circulado a ideia de fundir as atribuições dos ACS e dos Agentes de Combate às Endemias (ACE), tornando-os um único tipo de profissional17.

A PNAB 2017 desenvolve a materialização dessa ideia e afirma que "as atividades específicas dos agentes de saúde (ACS e ACE) devem ser integradas"3(73) sob o argumento da necessidade de união entre $\mathrm{a} A B$ e a vigilância em saúde, visando ao sucesso das ações desenvolvidas nos territórios. Em seguida, define as atribuições que devem ser comuns a ambos os agentes e as atribuições específicas de cada um.

Nessa distribuição, percebem-se três movimentos. O primeiro é a inclusão, na lista de atribuições comuns aos ACS e aos ACE, das atividades historicamente destinadas aos ACS, tais como: o desenvolvimento de ações de promoção da saúde e prevenção de agravos e riscos; a realização de visitas domiciliares periódicas para o monitoramento da situação de saúde das pessoas; a identificação e o registro de situações de risco à saúde; e a orientação, a informação e a mobilização da comunidade. O segundo movimento é a predominância, na lista das atribuições específicas dos ACS, de atividades de produção e registro de uma série de dados e informações pelas quais somente este trabalhador é responsável. O terceiro corresponde à manutenção do trabalho atualmente já 
desenvolvido pelos ACE na lista das atribuições específicas desse trabalhador.

Em síntese, o ACE preserva a responsabilidade sobre práticas tradicionalmente associadas ao seu trabalho, mas sofre um acréscimo em suas tarefas ao incorporar as atividades hoje atribuídas aos ACS. Estes, por sua vez, sofrem uma descaracterização do seu trabalho, que já vem sendo esvaziado das ações de educação em saúde, em virtude da priorização de atividades associadas às linhas de cuidado', que têm assumido um foco na prevenção de doenças, assim destinando a esses trabalhadores ações mais pontuais.

É preciso também considerar o quanto a integração entre esses profissionais estaria mais a serviço do corte de custos, pela diminuição de postos de trabalho, do que do aprimoramento do processo de trabalho e do aumento da eficiência das EqSF. Um resultado provável para esta relação será uma intensificação ainda maior do trabalho dos agentes que restarem nas equipes.

Ainda que se concorde com a necessidade de aproximação e de articulação entre os campos da vigilância e da atenção à saúde, isto não se resume à fusão de seus agentes. Enquanto ainda não se efetivaram esforços suficientes para se alcançar a integração dos ACE nas EqSF, conforme estabelecido pela PNAB de $2011^{2}$, projeta-se um processo de trabalho que desconsidera as especificidades dessas áreas técnicas e suas respectivas políticas. Além disto, não se desenvolvem pontos de integração no planejamento do trabalho e da formação, nem projetos de ação conjunta entre esses trabalhadores, nos territórios e nas unidades de saúde.

\section{Fragilização da coordenação nacional no pacto federativo da saúde}

O SUS possui caráter nacional e universal, e integra um conjunto amplo de ações que devem ser organizadas em uma rede descentralizada, regionalizada e hierarquizada de serviços. Vários aspectos influenciam a implantação deste modelo, entre eles, as características da federação brasileira, que se distingue pelo reconhecimento dos municípios como entes autônomos a partir da Constituição de 1988. Tais municípios (5.570, no total) são muito desiguais entre si e, em sua maioria (cerca de 90\%), possuem pequeno ou médio porte populacional e limitadas condições político-institucionais para assumirem as responsabilidades sobre a gestão das políticas de saúde que lhes são atribuídas.

Diversos foram os esforços empreendidos na trajetória de implantação do SUS para lidar com as tensões do federalismo brasileiro. As políticas direcionadas para o fortalecimento da ESF permitiram a consolidação do papel coordenador da União no pacto federativo da saúde ${ }^{22}$. Normas e incentivos financeiros federais favoreceram a implantação das políticas pelos municípios, com desconcentração de serviços no território e consolidação da ESF como referência nacional para organização da AB no SUS. Essas políticas também possibilitaram uma redistribuição não desprezível de recursos financeiros, com privilegiamento de municípios situados em regiões mais carentes, mesmo em um contexto de dificuldades de financiamento da saúde 23 .

A nova PNAB, ao flexibilizar o modelo de atenção e do uso dos recursos transferidos por meio do $\mathrm{PAB}$ variável, fragiliza o poder de regulação e indução nacional exercido pelo MS, responsável por avanços significativos no processo de descentralização do SUS. No que tange ao financiamento, ressalta-se a ausência de mecanismos de valorização diferenciada da ESF em relação às chamadas equipes de $\mathrm{AB}$, para as quais, anteriormente, não eram destinados esses recursos.

O fato é que passa a ser facultada à gestão municipal a possibilidade de compor equipes de $\mathrm{AB}$, de acordo com as características e as 
necessidades do município. Mais uma vez, aparece o recurso às especificidades locais como justificativa para a flexibilização do modelo de AB. No discurso dos gestores, a abertura de 'novas' possibilidades de financiamento e organização da $\mathrm{AB}$ tem sido valorizada quase como um reparo às supostas 'injustiças' cometidas contra as formas tradicionais de configuração da AB. O MS justifica as mudanças alegando que as regras praticadas nas PNAB anteriores acarretaram um desfinanciamento de parte dos serviços de $\mathrm{AB}$ existentes no País.

Entretanto, esses argumentos mostram-se falaciosos na medida em que suprimem do debate as análises que mostram os impactos positivos da adoção e expansão da ESF nas condições de vida e saúde da população. Também não levam em consideração que as especificidades locorregionais já eram objeto das PNAB anteriores e de incentivos financeiros vigentes, sendo possível observar a adoção de adaptações ao modelo preconizado pela ESF em vários municípios do País. Além disto, tais mudanças acabam por favorecer as capitais e os centros urbanos metropolitanos, que aderiram à ESF de modo tardio e apresentam menor cobertura. Isso poderá acarretar um redirecionamento de recursos para regiões mais desenvolvidas e com maior capacidade de arrecadação de tributos, comprometendo os avanços alocativos do setor.

Por fim, mais uma vez, chama a atenção a ausência de uma análise contextualizada sobre o impacto das mudanças que estão sendo propostas na PNAB 2017, e que serão implementadas nos próximos anos. A liberdade e a maior autonomia requeridas pelos gestores locais se inserem em uma conjuntura de ameaças aos direitos sociais, e de forte restrição fiscal e orçamentária, com agravamento da situação de subfinanciamento do SUS. Esse contexto condicionará as escolhas políticas, ampliando as dificuldades locorregionais para a manutenção de serviços de $\mathrm{AB}$ frente aos custos elevados da atenção de média e alta complexidade no SUS. A escassez de recursos públicos disponíveis para a saúde também tenderá a aumentar as disputas redistributivas, favorecendo a influência de interesses particulares nas decisões alocativas do setor, a implantação de planos privados de cobertura restrita e de modelos alternativos à ESF, com resultados duvidosos para a organização da $\mathrm{AB}$.

\section{Considerações finais}

Desde o início dos anos 1990, para grande parte da população brasileira, a $\mathrm{AB}$ tem sido a face mais notável de um sistema de saúde orientado por princípios de universalidade, integralidade e equidade. Confrontada com tendências que priorizam programas focalizantes e compensatórios, mais afeitos à racionalidade neoliberal hegemônica, a ESF tem se configurado como meio de expansão do acesso e de realização do direito à saúde.

Alçada à condição de estratégia de reorientação do modelo de atenção à saúde, tem ainda um longo caminho a percorrer e obstáculos a ultrapassar, antes que seja alcançado o objetivo de efetivar uma clínica ampliada, que articule diferentes saberes, trabalhadores e setores da política pública, de modo a compreender e enfrentar os determinantes sociais do processo saúde-doença.

Há muito a ser reorganizado e modificado em função dos resultados das pesquisas e das avaliações realizadas sobre a AB. A qualificação de trabalhadores de todos os níveis de formação que compõem as EqSF, visando ao trabalho baseado no território; a integração entre prevenção, atenção e promoção da saúde; a organização de processos de trabalho mais democráticos e participativos, nos quais os trabalhadores tenham papel importante no planejamento das ações, na definição e discussão das metas e prioridades das equipes; a gestão pública do trabalho e dos serviços de $\mathrm{AB}$; e a regularização dos vínculos contratuais são questões que precisam ser priorizadas. 
Entretanto, as modificações introduzidas na PNAB 2017 apontam para outra direção. Em um breve inventário, destacam-se alguns mecanismos da nova política que promovem a relativização da cobertura universal, a definição de padrões distintos de serviços, a recomposição das equipes e a reorganização do processo de trabalho na $\mathrm{AB}$, entre outras mudanças importantes, que também devem ser consideradas. A revisão empreendida atinge principalmente as conquistas alcançadas pela ESF e os processos em curso, que pavimentam caminhos para se concretizar uma APS forte, parte fundamental de redes de atenção em um sistema universal de atenção integral à saúde.

Em nome de uma suposta maior autonomia dos municípios, o MS renuncia à sua responsabilidade de coordenação e indução de bases nacionais para a PNAB, fato bastante arriscado em um país com realidades locorregionais tão distintas e com um processo de descentralização que carece de aprimoramentos. São particularmente preocupantes as consequências do possível descompromisso dos gestores com a oferta universal dos serviços de AB; a segmentação do acesso ao cuidado; a desvinculação das equipes dos territórios; e a desqualificação do trabalho de ACS e ACE, acentuando o caráter utilitarista de suas atividades e o reforço à privatização.
Estas questões articuladas agravam o risco de desassistência de parte significativa da população, e de perda na qualidade dos serviços da AB. Tais ameaças tornam-se especialmente graves em um momento de ruptura das relações e instituições democráticas, e de naturalização de um processo crescente de subtração de direitos e aprofundamento da desigualdade. Consolida-se um processo anticivilizatório do capital, que precisa mercantilizar cada vez mais espaços de produção e reprodução da vida humana. Desassistir frações da classe trabalhadora com alguma capacidade de comprar serviços de saúde como parece ser o horizonte desenhado pela PNAB 2017 - significa que o Estado brasileiro atua, mais uma vez, em favor da ampliação da participação do setor privado na saúde, em detrimento de um sistema que nunca pôde ser inteiramente público.

Em contraposição, como projeto de luta e transformação, defende-se que os princípios do SUS, democraticamente constituídos e legalmente estabelecidos, deveriam ser tomados como valores éticos, que precedem e orientam a reconfiguração das políticas de saúde. Portanto, não devem e não podem ser transgredidos ou sequer flexibilizados, seja em função da tão aludida crise econômica vigente, muito menos em atenção às necessidades do mercado. 


\section{Referências}

1. Brasil. Ministério da Saúde. Portaria n ${ }^{\circ} 648$, de 28 de março de 2006. Dispõe sobre a revisão de diretrizes e normas para a organização da Atenção Básica para o Programa da Saúde da Família (PSF) e o Programa de Agentes Comunitários de Saúde (Pacs). Diário Oficial da União. 2006 Mar 28. [acesso em $2018 \mathrm{fev}$ 27]. Disponível em: http://bvsms.saude.gov.br/bvs/ saudelegis/gm/2006/prt0648_28_03_2006_comp. html.

2. Brasil. Ministério da Saúde. Portaria $\mathrm{n}^{\circ}$ 2.488, de 21 de outubro de 2011. Aprova a Política Nacional de Atenção Básica, estabelecendo a revisão de diretrizes e normas para a organização da Atenção Básica, para a Estratégia Saúde da Família (ESF) e o Programa de Agentes Comunitários de Saúde (Pacs). Diário Oficial da República Federativa do Brasil. Brasília, DF, 22 Out. 2011. [acesso em 2017 nov 22]. Disponível em: http://bvsms.saude.gov.br/ bvs/saudelegis/gm/2011/prt2488_21_10_2011.html.

3. Brasil. Ministério da Saúde. Portaria no 2.436, de 21 de setembro de 2017. Aprova a Política Nacional de Atenção Básica, estabelecendo a revisão de diretrizes para a organização da Atenção Básica, no âmbito do Sistema Único de Saúde (SUS). Brasília, DF: Ministério da Saúde, 2017. [internet]. [acesso em 2017 nov 30]. Disponível em: http://www.brasilsus.com.br/index.php/legislacoes/gabinete -do-ministro/16247-portaria-n-2-436-de-21-de-setembro-de-2017.

4. Giovanella L, Mendonça MH. Atenção primária à saúde. In: Giovanella L, Escorel S, Lobato LVV, et al., organizadores. Políticas e Sistema de Saúde no Brasil. Rio de Janeiro: Ed. Fiocruz; 2012. p. 493-545.

5. Castro ALB, Fausto MCR. A política brasileira de atenção primária à saúde. In: Machado CV, Baptista TWF, Lima LD, organizadores. Políticas de saúde no Brasil: continuidades e mudanças. Rio de Janeiro: Editora Fiocruz; 2012. p. 173-196.

6. Abrasco; Cebes; Ensp. Contra a reformulação da PNA - nota sobre a revisão da Política Nacional de
Atenção Básica. [internet]. [acesso em 2018 fev 22]. Disponível em: https://www.abrasco.org.br/site/ noticias/posicionamentos-oficiais/contra-reformulacao-da-pnab-nota-sobre-revisao-da-politica-nacional-de-atencao-basica/29798/.

7. Levcovitz E, Lima LD, Machado CV. Política de saúde nos anos 90: relações intergovernamentais e papel das normas operacionais básicas. Ciênc. Saúde Colet., 2001; 6(2):269-91.

8. Brasil. Ministério da Saúde. Portaria $\mathrm{n}^{0} 3.925$, de 13 de novembro de 1998. Aprova o Manual para a Organização da Atenção Básica no Sistema Único de Saúde. [internet]. [acesso em 2017 dez 10]. Disponível em: http://bvsms.saude.gov.br/bvs/saudelegis/gm/1998/prt3925_13_11_1998_rep.html.

9. Fonseca AF, Mendonça MH. Avaliação e políticas públicas de saúde: reflexões a partir da atenção básica. In: Guizardi F, Nespoli G, Cunha ML, et al., organizadores. Políticas de Participação e Saúde. led. Recife: Editora Universitária-UFPE, 2014, p. 197-224.

10. Brasil. Ministério da Saúde. Secretaria Executiva. Coordenação de apoio à gestão descentralizada. Diretrizes operacionais para os pactos pela vida, em defesa do SUS e de gestão. Brasília, DF: Ministério da Saúde, 2006b. [internet]. [acesso em 2017 dez 01]. Disponível em: http://www.saude.mppr.mp.br/ arquivos/File/volumel.pdf.

11. Magalhães Jr HM, Pinto HA. Atenção básica enquanto ordenadora da rede e coordenadora do cuidado: ainda uma utopia? Divulg. Saúde debate [internet]. 2014 Out; 51:14-29. [acesso em $2018 \mathrm{fev}$ 20]. Disponível em: http://cebes.org.br/site/wp-content/uploads/2014/12/Divulgacao-51.pdf.

12. Silva EN, Powell-Jackson T. Does expanding primary healthcare improve hospital efficiency? Evidence from a panel analysis of avoidable hospitalisations in 5.506 municipalities in Brazil, 2000-2014. BMJ Global Health 2017; 2:e000242. 
13. Pereira AMM, Castro ALB, Malagón Oviedo RA, et al. Atenção primária à saúde na América do Sul em perspectiva comparada: mudanças e tendências. Saúde debate [internet] 2012 Set; [acesso em $2017 \mathrm{dez}$ 3]; 36(94):482-499. Disponível em: http://www.scielo.br/scielo.php?script=sci arttext\&pid=S0103-11042012000300019\&lng=pt\&n rm=iso.

14. Giovanella L, Almeida PF, Romero RM, et al. Panorama de la Atención Primaria de Salud en Suramérica: concepciones, componentes y desafíos. Saúde debate, 2015 Jun; 39(105):300-322.

15. Ocké-Reis CO. Desafios da reforma sanitária na atual conjuntura histórica. Saúde debate [internet]. 2017 Abr [acessoem 2017 dez1];41(113);365-371.Disponível em: $\quad$ http://www.scielo.br/scielo.php?script=sci arttext\&pid=S0103-11042017000200365\&lng=pt\&nr $\mathrm{m}=$ iso.

16. Brasil. Ministério da Saúde. Secretaria de Atenção à Saúde Departamento de Atenção Básica. VII Fórum Nacional de Gestão da Atenção Básica. [internet]. [acesso em 2016 nov 30]. Disponível em: https:// blog.atencaobasica.org.br/wp-content/uploads/2016/10/Síntese-VII-Fórum-AB.pdf.

17. Morosini MVGC, Fonseca AF. Revisão da Política Nacional de Atenção Básica numa hora dessas? Cad. Saúde Pública [internet]. 2017 [acesso em $2017 \mathrm{dez}$ 1]; 33(1):e00206316. Disponível em: http://www. scielo.br/scielo.php?scrip t=sci_arttext\&pid=S0102 $=-311 X 2017000100301 \& \operatorname{lng}=$ pt\&nrm=iso.
18. Conill EM, Fausto MCR, Giovanella L. Contribuições da análise comparada para um marco abrangente na avaliação de sistemas orientados pela atenção primária na América Latina. Rev. Bras. Saúde Matern. Infant. 2010; 10(Supl.1):15-27.

19. Silva SF. Organização de redes regionalizadas e integradas de atenção à saúde: desafios do Sistema Único de Saúde (Brasil). Ciênc. Saúde Colet. 2011; 16(6):2753-2762.

20. Empresa Brasileira de Comunicação. Plano de saúde mais barato aliviará gasto com o SUS, diz Barros. Por Flávia Albuquerque 2016 Ago 8. [internet]. [acesso em 2017 out 27]. Disponível em: http:// agenciabrasil.ebc.com.br/geral/noticia/2016-08/ plano-de-saude-mais-barato-aliviara-gasto-com-financiamento-do-sus-diz-barros.

21. Barros R. Palestra proferida no seminário "Novos Modelos para a Saúde”. Realização O Globo. 2017 Ago 15; Museu de Arte do Rio, Rio de Janeiro.

22. Viana ALA, Machado CV. Descentralização e coordenação federativa: a experiência brasileira na saúde. Ciênc. Saúde Colet. 2009; 14(3):807-817.

23. Lima LD. Federalismo fiscal e financiamento descentralizado do SUS: balanço de uma década expandida. Trab. Educ. Saúde, 2009; 6(3):573-597.

\footnotetext{
Recebido em 06/12/ 2017

Aprovado em 01/03/2018

Conflito de interesses: inexistente

Suporte financeiro: não houve
} 